Arteterapia. Papeles de arteterapia y educación para inclusión social ISSN-e 1988-8309

http://dx.doi.org/10.5209/ARTE.57563

\title{
Un modelo estructural sobre la reducción del estrés utilizando material artístico
}

\author{
Minerva Vanegas-Farfano ${ }^{1}$, Mónica Teresa González Ramírez²; René Landero Hernández ${ }^{3}$
}

Recibido: 26 de abril de 2017 / Aceptado: 27 de julio de 2017

Resumen. En este trabajo se analiza el uso de actividades artísticas (dibujo) como una forma de regulación del estrés psicológico y las emociones asociadas. Para ello 216 alumnos de una facultad de Odontología del norte de México, participaron en un estudio bajo una de seis condiciones experimentales, donde recordaron y trabajaron dibujos relacionados a sus estresores actuales o se distrajeron de estos. Se utilizaron la Escala de Estrés Percibido, la Escala de Afecto Positivo y Negativo y el cuestionario la actividad gráfica como herramienta. Los resultados confirman la reducción del estrés percibido asociado al uso de la actividad gráfica y una consecuente disminución de las emociones negativas al término de una única sesión en todas las condiciones experimentales.

Palabras Clave: estrés percibido; actividad artística; regulación emocional; modelo estructural.

\section{[en] A structural model on stress reduction using artistic material}

Abstract. This work analyzes the use of artistic activities (drawing) as a form of regulation of psychological stress and the emotions that are associated with it. To this end, 216 students from a dental school in northern Mexico participated in a study under one of six experimental conditions, where they were sked to recall and work on drawings related to their current stressors or distract from them. The Perceived Stress Scale, the Positive and Negative Affect Scale and a questionnaire related to graphic activities used as tools were implemented. The results confirm the reduction of the perceived stress associated to the use of the graphic activity and a consequent decrease of the negative emotions at the end of a single session in all the experimental conditions.

Key Words: perceived stress; artistic activity; emotion regulation; structural model.

Cómo citar: Vargas-Farfano, M.; González Ramírez, M.T.; Ladero Hernández, R. (2017). Un modelo estructural sobre la reducción del estrés utilizando material artístico, en Arteterapia. Papeles de arteterapia y educación para inclusión social 12, 79-94.

\footnotetext{
Minerva Vanegas-Farfano. Doctora en Filosofía con Orientación en Psicología, UANL. Miembro del Sistema Nacional de Investigadores (Nivel 1). Universidad Autónoma de Nuevo León, UANL;

Email: minerva.vanegas@gmail.com

2 Mónica Teresa González Ramírez. Doctora en Metodología de las Ciencias del Comportamiento UAM, UNED, UCM. Miembro del Sistema Nacional de Investigadores (Nivel 2).

Email:

3 René Landero Hernández. Doctorado en Filosofía con especialidad en Trabajo Social y Políticas, UANL. Miembro del Sistema Nacional de Investigadores (Nivel 2).

Emal:
} 


\section{Introducción}

El estrés se incluye como uno de los fenómenos, en conjunto a la ansiedad, en donde existe una mayor posibilidad de eficacia del arte terapia (Slayton, D'Archer \& Kaplan, 2010). Con una vasta referencia dentro de investigaciones tanto cuantitativas como cualitativas (Bitonte \& De Santo, 2014; Drake \& Hodge, 2015; Drake, Coleman \& Winner, 2011; Hacking, Secker, Spandler, Kent \& Shenton, 2008), se puede incluso considerar como un aspecto psicológico modificable mediante este tipo de intervención. A pesar de ello, la forma como sucede este proceso no es explorado.

En este documento, se contrasta un modelo teórico, mediante ecuaciones estructurales, en torno a la reducción del estrés, gracias a la intervención con material artístico. Para ello, se hace brevemente una revisión cronológica del material publicado en torno al estrés percibido y su reducción por medio del arte, ya sea como variable principal o secundaria, y desde distintos métodos de estudio. En un segundo apartado, se presentan los mediadores relacionados y mecanismos asociados, para con ello dar pauta a la explicación de cómo estos fueron experimentalmente evaluados a fin de explicar el fenómeno observado.

\section{Efectos de la actividad artística en la reducción del estrés: un estudio de más de dos décadas}

El crear, como proceso, se ha comprobado como una actividad que alivia el estrés mediante distintas formas y técnicas (Malchiodi, 2005). En revisiones y meta análisis rigurosos figura como uno de los afectos que, tanto en niños como adolescentes y adultos, se reduce, al mismo tiempo en que apoya el afrontar otros sentimientos (Reynolds, Nabors \& Quinlan, 2000; Slayton et al., 2010).

La incursión del arte dentro del ambiente de la salud ha tenido como uno de sus objetivos el apoyar el alivio desde medios menos invasivos que la palabra; el estudio de los efectos del arte para el manejo del estrés abarca desde las manifestaciones más graves, como es el estrés post traumático (Sarid \& Huss, 2010), hasta las menos agudas, como el manejo del estrés cotidiano (Holmqvist \& Lundqvist, 2012; Kim, 2013; Vanegas-Farfano, Quezada-Berumen \& González Ramírez; 2016), llegando incluso a la popularización de la técnica.

En cuanto a su formato, ya sea en torno a situaciones no habituales, como tener que lidiar con una enfermedad crónica o terminal, por ejemplo, el cáncer (Mische, et al., 2012); o bien centrándose en lo cotidiano, como es reconocer situaciones estresantes habituales en el lugar de trabajo (Mercer, Warson \& Zhao, 2010; Visnola, Sprudza, Bake \& Pike, 2010) o la escuela (Regehr, Glancy \& Pitts, 2013), la actividad artística se presenta como una opción con amplia gama de técnicas, herramientas y actividad a realizar, tanto en lo solitario como en grupo, con o sin la intervención de un terapeuta.

Desde un punto de vista metodológico, el efecto de las actividades artísticas, para lidiar con el estrés se ha revisado tanto fuera, como dentro de espacios experimentales. Desde este último se ha trabajado su reducción tras la inducción mediante estímulos adversos como películas (Drake, Coleman \& Winner, 2011) e incluso trabajando con los propios estresores (Vanegas-Farfano, Quezada-Berumen \& González Ramírez; 2016). Mediante este tipo de investigaciones, se ha logrado aislar y 
corroborar los distintos elementos que están en juego durante este proceso: se sabe que existen distintas formas para manejar los estresores, los cuales se orientan a la solución del problema que lo origina o el dejar de atenderle (Aydin, 2010; Drake \& Winner 2012a); y que esto se encuentra arraigado en las primeras formas de afrontamiento desarrolladas desde la infancia (Drake \& Winner, 2012b).

Si bien lo anterior nos permite saber que el fenómeno en si existe -la actividad artística reduce el estrés psicológico- y que esto se relaciona con al menos dos formas de afrontamiento equidistantes; el estudio enfocado a los agentes que le promueven es escaso desde una perspectiva metodológica cuantitativa, lo que incide en la su promoción como intervención única. Siendo necesario por ello, indagar en el fenómeno más allá de lo anecdótico. La siguiente sección, retoma las explicaciones dadas en torno al qué y por qué, un agente determinado llega a contribuir con la reducción del estrés al ser utilizado desde lo artístico. Para ello, y por motivos metodológicos a explicarse en la siguiente sección, la información a la que se hace referencia se concentra únicamente en uso del material gráfico.

\section{Arte y estrés: mediadores y mecanismos relacionados.}

El manejo del estrés mediante material artístico incluye tanto procesos cognitivos como emocionales (Drake \& Winner, 2012a; Drake \& Winner, 2012b). La actividad artística reduce el estrés de manera activa cuando involucra funciones cognitivas como las representaciones mentales. Desde esta perspectiva, la actividad artística permite una reorganización de los pensamientos o un enfoque en otros incompatibles a los estresores (Dilawari \& Tripathi, 2014). Evidencia de ello son los estudios en donde se da la oportunidad para expresar y proponer respuestas a los estresores (Huss, Nuttman-Shwartze \& Altman 2012); o, por otro lado, dar pie a un espacio de tranquilidad al considerarle una distracción (Reynolds, 2010; Singer, Götze, Buttstädt, Geue, Momenghalibaf \& Böhler, 2010). En cuanto a las emociones, la actividad artística posibilita un alivio de estas al expresarlas (Huet \& Holttum, 2016). Las emociones, al coexistir en una relación parte-todo, con el estrés (Lazarus, 2000), permiten por su estudio identificar que el predominio de las emociones negativas predispone al estrés (O’Brien, Terry \& Jimmieson, 2008). Se sabe además que las emociones positivas apoyan al prolongar su efecto por cierto periodo de tiempo una vez terminado el evento que las origina (Fredrickson \& Joiner, 2002). Por último, dado que las actividades artísticas se acompañan del trabajo de un terapeuta se agregó a este estudio una actividad -desarrollo de texto- que asemejara la elaboración realizada frente a este al momento de trabajar en sesión.

A pesar de encontrarse considerable documentación sobre la evaluación de la actividad artística, concentrada en el estrés y las emociones, desde su introducción al ámbito de la salud un aspecto poco estudiado desde métodos cuantitativos es la especificidad de la técnica. Con una mayor concentración en el estudio de su controlabilidad y criterios a seguir dependiendo del tipo de afecciones de los pacientes, por ejemplo, propensión al psicoticismo (Marxen, 2011); la investigación de Snir y Regev (2013), se coloca como una de las pocas que incluye en su revisión aspectos como las actitudes hacia lo creado, el proceso y el acercamiento hacia el material. En este sentido, cabe enfatizar estudios como el de Huet y Holttum (2016) que consideran que, el utilizar a la actividad artística como herramienta para manejar los 
estresores, coexiste la preocupación inicial sobre las habilidades necesarias para el uso de los materiales, seguido de un creciente sentimiento de que ello es superable a la vez que disfrutable.

\section{Una propuesta del arte como elemento mediador}

El presente estudio pretende profundizar en el análisis del manejo del estrés percibido y las emociones, a través de actividades artísticas. Para lograrlo, su diseño toma como referencia los resultados de estudios previos, en cuanto a que una intervención de esta naturaleza habrá de incluir tanto elementos cognitivos -qué se hará con el material-, como emocionales -qué se expresa con ello-; y una necesaria revisión antes y después de dicha intervención. Fue por ello considerado como apropiado que ambos aspectos, cognitivo (percepción) y emoción, sean desde el método variantes, grupos experimentales, a través de las instrucciones dadas a los participantes. Mientras que la relación que mantienen ambos aspectos psicológicos con el uso del material se posicione como un mediador, el cual habrá de incluir la actitud hacia lo creado, el proceso y el acercamiento al material. En otras palabras, esta investigación plantea el desarrollo de un modelo explicativo de los cambios observados tras una sesión de actividad artística, contemplando la interacción de dichos elementos.

Por último, considerando los estudios ya señalados, hipotetizamos que: a) predominará la disminución de las emociones negativas frente al cambio en las emociones positivas, y b) los efectos observados se vincularan tanto al enfoque como a la distracción de los estresores.

\section{Método}

\section{Participantes}

La muestra se compuso por 216 estudiantes de odontología (53 hombres y 162 mujeres) de una misma universidad; con un rango de edad que oscilaba entre los 17 a los 22 años (media=19.88; D.E.=4.17). El muestreo fue aleatorio estratificado, contemplando cada semestre como estrato con la finalidad de tener una muestra representativa de toda la población de la facultad. Es decir, se contó con la participación de estudiantes de primero a noveno semestre en cada grupo experimental.

Se manejaron como criterios de inclusión que el alumno se encontrase inscrito en al menos la mitad de las materias consideradas como carga completa, que se sintiese capacitado para realizar actividades gráficas (dibujo) a nivel no profesional y que pudiese identificar una situación estresante que influya en ese momento en su vida. Fueron excluidos aquellos alumnos que se encontraran en periodo de duelo o presentasen la confirmación de un diagnóstico de ansiedad, depresión o estrés post-traumático y/ un problema de abuso de sustancias.

\section{Instrumentos y materiales}

Se proporcionó a los participantes un sobre cerrado que contenía: 
- La Escala de Estrés Percibido (PSS) elaborada por Cohen, Kamarck y Mermelstein, en 1983 y adaptada en México por González y Landero (2007). Dicha escala tiene catorce ítems con puntuación de nunca (0) a muy a menudo (4), con coeficiente de fiabilidad de .83 y datos normativos para población mexicana por sexo y por grupo etario (González-Ramírez, Rodríguez-Ayán \& Landero-Hernández, 2013).

- La escala de Afecto Positivo y Negativo (PANAS, Watson, Clark \& Tellegen, 1988) en su adaptación para población mexicana por Robles y Páez (2003). Esta escala mide las dos dimensiones de emocionalidad, afecto positivo y negativo. Incluye veinte reactivos divididos en dos apartados (diez para medir el afecto positivo y diez para el afecto negativo. Dicha versión valora los sentimientos y emociones en la última semana, y cuenta con una fiabilidad interna de .85 a. 81

- Un cuestionario sobre el empleo de la actividad gráfica como herramienta $(A G H)$ cuyo objetivo es el auto-reporte del proceso creativo experimentado en la sesión. Dicho instrumento incluyó 17 ítems, once de ellos provenientes del Art-based Intervention Questionnaire de Snir \& Regev, (ABI; por sus siglas en inglés, 2013); los ítems de dicha escala se configuraron en tres dimensiones: estrategia centrada en la solución de problemas, placer y valor terapéutico y competencia y uso del material.

Además, los materiales artísticos empleados consistieron en a) una hoja tamaño carta de papel fabriano natural de bajo gramaje, b) un paquete de crayones suaves tipo pastel de 12 piezas y c) lápiz con borrador y material para limpieza.

- Como parte del debrief se integró una Actividad vs. Emociones negativas. La cual se diseñó para eliminar residuos de emociones negativas mediante la enumeración de estrategias, recursos o posibles desenlaces positivos de acuerdo a habilidades actuales de los participantes.

En el caso de aquellos participantes que aleatoriamente trabajaron bajo la instrucción pasiva (ver sección instrucciones) se emplearon imágenes del International Affective Picture System (IAPS) de Lang, Bradley y Cuthbert (2008). Las imágenes incluidas proporcionaban entornos e interiores urbanos neutras de acuerdo a sus medias y desviaciones estándar en las variables de placer, estimulación y dominancia.

\section{Diseño}

Como ya se señaló, a fin de conocer la relación entre las variables de estudio bajo una serie de instrucciones ya señaladas como observables, esta investigación se desarrolló mediante un diseño experimental con seis grupos de intervención y medidas repetidas, antes y después de la ejecución de la actividad gráfica. La tabla 1 presenta la relación de los grupos (GRUPO) con la instrucción dada (Tratamiento) y la relación de los cuestionarios utilizados en cada uno de ellos. La "X" señala el momento en que fue empleada la actividad gráfica. La estratificación realizada fue por semestre y permitió una distribución casi homogénea entre grupos, dato que se observa en esa misma tabla. 
Tabla 1

Tratamiento por grupo experimental (seis grupos)

\begin{tabular}{|l|l|l|l|l|}
\hline GRUPO & Tratamiento & \multicolumn{2}{|l|}{ Diseño } \\
\hline $\begin{array}{l}a_{1} \\
(\mathrm{n}=36)\end{array}$ & $\begin{array}{l}\text { Actividad como distractor activo }+ \text { Narra- } \\
\text { tiva de suceso }\end{array}$ & $\mathrm{E}_{1}, \mathrm{P}_{1}, \mathrm{C}$ & $\mathrm{X}$ & $\mathrm{E}_{2}, \mathrm{P}_{2}, \mathrm{G}, \mathrm{A}$ \\
\hline $\begin{array}{l}a_{2} \\
(\mathrm{n}=36)\end{array}$ & $\begin{array}{l}\text { Actividad como distractor activo }+\mathrm{Sin} \\
\text { narrativa del suceso }\end{array}$ & $\mathrm{E}_{1}, \mathrm{P}_{1}$ & $\mathrm{X}$ & $\mathrm{E}_{2}, \mathrm{P}_{2}, \mathrm{C}, \mathrm{G}, \mathrm{A}$ \\
\hline $\begin{array}{l}a_{3} \\
(\mathrm{n}=36)\end{array}$ & $\begin{array}{l}\text { Actividad como distractor pasivo }+\mathrm{Na}- \\
\text { rrativa de suceso }\end{array}$ & $\mathrm{E}_{1}, \mathrm{P}_{1}, \mathrm{C}$ & $\mathrm{X}$ & $\mathrm{E}_{2}, \mathrm{P}_{2}, \mathrm{G}, \mathrm{A}$ \\
\hline $\begin{array}{l}a_{4} \\
(\mathrm{n}=39)\end{array}$ & $\begin{array}{l}\text { Actividad como distractor pasivo }+\mathrm{Sin} \\
\text { narrativa del suceso }\end{array}$ & $\mathrm{E}_{1}, \mathrm{P}_{1}$ & $\mathrm{X}$ & $\mathrm{G}_{2}, \mathrm{E}_{2}, \mathrm{P}_{2}, \mathrm{C}, \mathrm{A}$ \\
\hline $\begin{array}{l}a_{5} \\
(\mathrm{n}=36)\end{array}$ & $\begin{array}{l}\text { Actividad como enfoque }+ \text { Narrativa de } \\
\text { suceso }\end{array}$ & $\mathrm{E}_{1}, \mathrm{P}_{1}, \mathrm{C}$ & $\mathrm{X}$ & $\mathrm{G}_{2}, \mathrm{E}_{2}, \mathrm{P}_{2}, \mathrm{~A}$ \\
\hline $\begin{array}{l}a_{6} \\
(\mathrm{n}=33)\end{array}$ & $\begin{array}{l}\text { Actividad como enfoque }+ \text { Sin narrativa } \\
\text { del suceso }\end{array}$ & $\mathrm{E}_{1}, \mathrm{P}_{1}$ & $\mathrm{X}$ & $\mathrm{G}_{2}, \mathrm{E}_{2}, \mathrm{P}_{2}, \mathrm{C}, \mathrm{A}$ \\
\hline
\end{tabular}

NOTA: (E) Escala de estrés percibido; (P) Escala de afecto positivo y negativo, PANAS; (C) Cuestionario de actividad gráfica como herramienta, (G) Componente cualitativo del CSI; (A) Actividad vs. Emociones negativas. Los subíndices 1 y 2 corresponden al tiempo (1: preintervención; 2: postintervención).

\section{Procedimiento}

Una vez contactadas las autoridades del plantel se procedió a invitar a los alumnos en las aulas, ahí se anunció verbalmente el objetivo de la actividad: se les indicaba que para participar se les solicitaría pensar en una situación actual que considerasen estresante y que, tras ello, se les pediría realizar un dibujo o un dibujo y un texto sobre este, de acuerdo con unas instrucciones asignadas de manera aleatoria. Al igual que cumplimentar dos juegos de cuestionarios, uno antes y el otro después de realizar las actividades. Los cuestionarios fueron aplicados de forma auto-administrada durante el periodo académico de agosto de 2015. Los estudiantes que participaron lo hicieron de manera voluntaria y anónima. Todos ellos fueron informados por escrito de su consentimiento, así como de los objetivos de la investigación junto con un paquete donde se incluían los cuestionarios a llenar debidamente ordenados (Tabla 1). Los sobres se identificaron con un color diferente para cada grupo experimental; como se señaló anteriormente se les notificaba las instrucciones de trabajo de acuerdo con el color asignado. La asignación por grupo experimental fue aleatoria. 
Instrucciones para los participantes (Tratamiento). Para este protocolo se trabajó con una instrucción orientada al uso del material gráfico, realizada por los seis grupos experimentales y otra encaminada a la realización de un texto, en ésta, sólo participaba la mitad de los grupos. Primero, con el objeto de que trabajasen sobre una situación estresante actual que pudiese ser similar a todos los participantes, se seleccionó la instrucción del componente cualitativo del Inventario de Estrategias de Afrontamiento (CSI por sus siglas en inglés), en su adaptación española realizada por Cano et al., 2007. Esta consigna se orienta a la descripción de un evento cuyas características permitan considerarlo como estresante: algo que causa problemas, haga sentirse mal o cuesta mucho enfrentarse a ella. Dicha situación debía formar parte de lo cotidiano, es decir, ser una situación de índole relacional, familiar o laboral. Segundo, se solicitaba a los participantes el trabajar el uso de la actividad gráfica como un distractor o una herramienta de enfoque en sus estresores, de acuerdo con la asignación aleatoria a cada grupo experimental y siguiendo las siguientes instrucciones:

a) Grupos en el rubro de distracción (cuatro grupos, dos trabajando bajo una condición de distracción pasiva y dos bajo una condición de distracción activa; Figuras 1 y 2, respectivamente): a los grupos bajo la condición de distracción pasiva se les indicaba "copiar una de las imágenes incluida en el material, sin importar que tan perfecto o bello quede el dibujo"; los grupos bajo la condición de distracción activa siguieron la instrucción: "representa un objeto o situación que te permita distraerte del estresor que acabas de pensar y/o representar".

b) Grupos en el rubro de enfoque (dos grupos; Figura 3): la instrucción dada estos grupos fue "representar el objeto o situación estresante que haya documento en los cuestionarios anteriores, sin importar que tan perfecto o bello quede el dibujo".

Tercero (instrucción orientada al uso del texto): Únicamente a tres grupos se les solicitaba el realizar una narración, uno de los grupos de distracción activa, uno de distracción pasiva y un tercero bajo el rubro de enfoque en el problema. Esta instrucción consistía en realizar una narración sobre la situación estresante que hayan seleccionado. En el caso de los participantes que no hicieron una redacción anterior al dibujo la instrucción fue: "representar el objeto o situación estresante que haya considerado como estresor actual, sin importar que tan perfecto o bello quede el dibujo".

Por último, dada la naturaleza experimental de este estudio su debrief consistió en la implementación de la Actividad vs. Emociones negativas, con todos los participantes. 


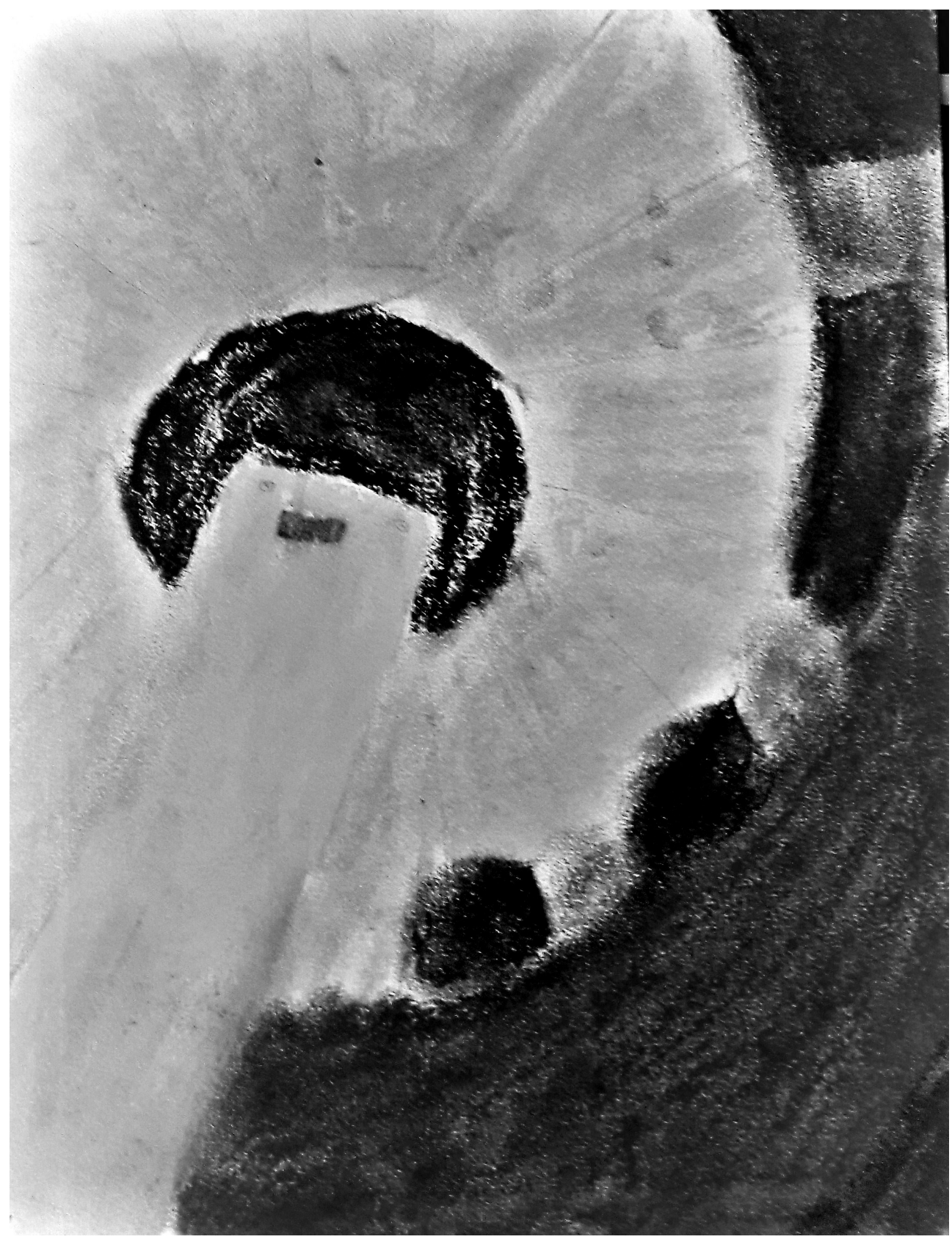




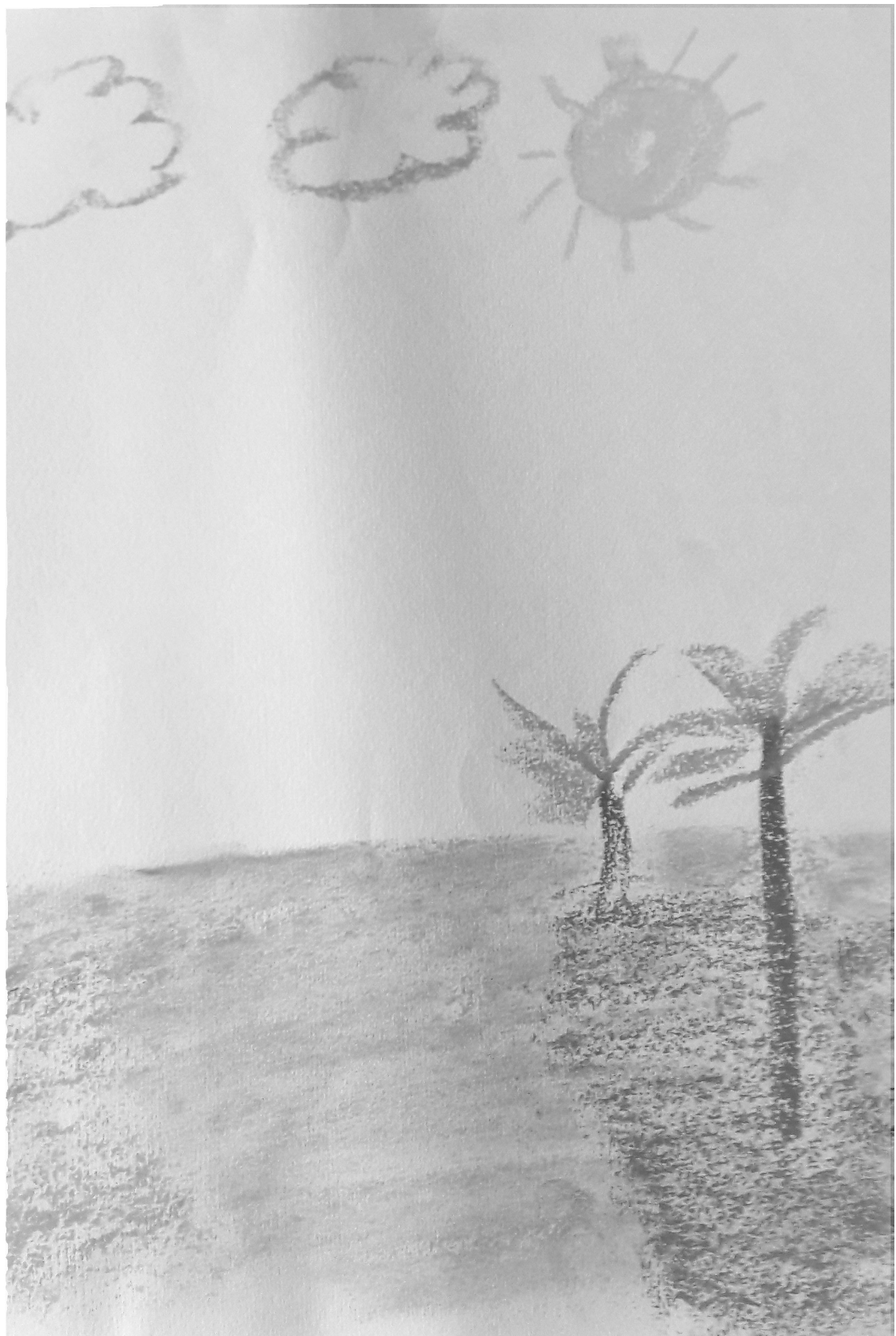




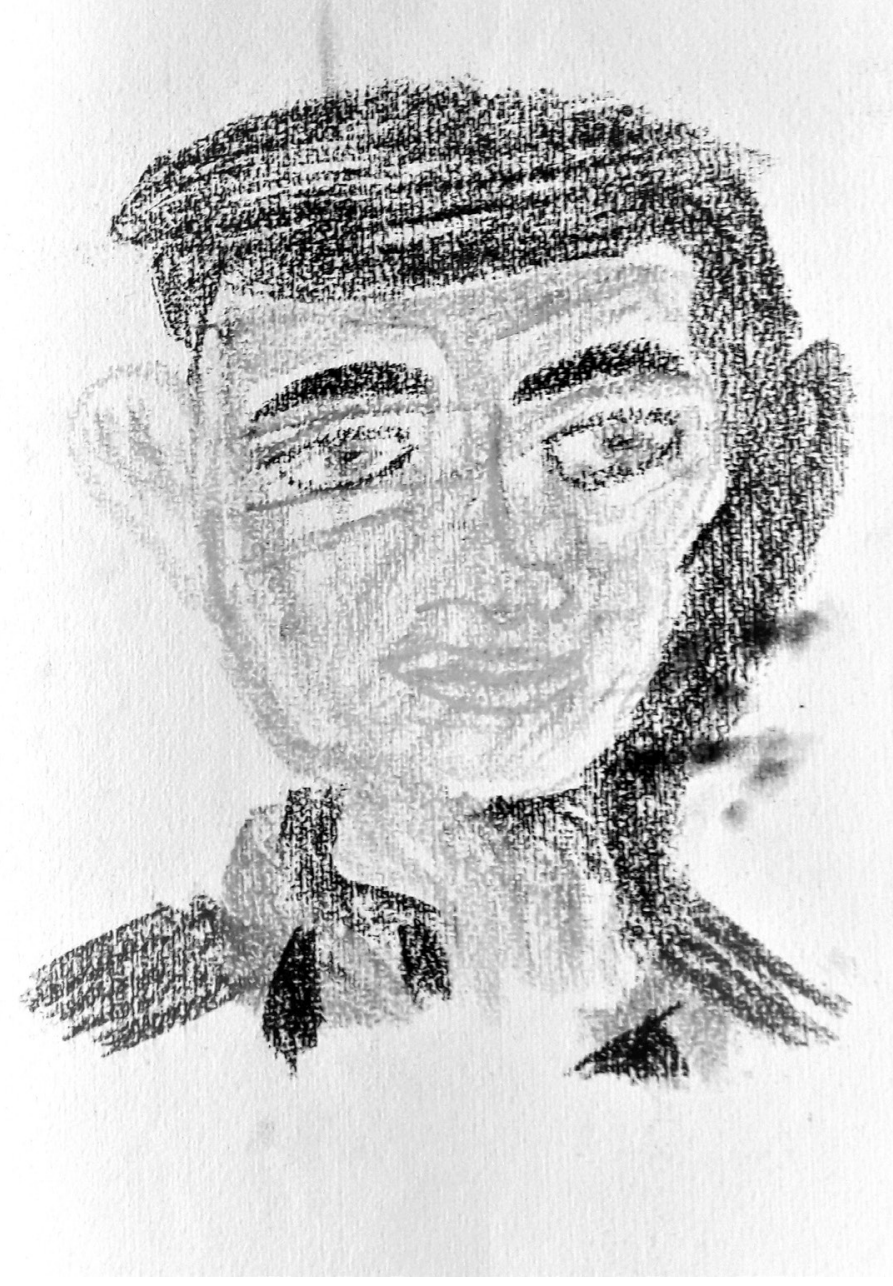

\section{Análisis}

Se analizaron los datos sociodemográficos mediante estadísticos descriptivos. También la confiabilidad de todas las escalas. Para comprobar que no hubiese diferencias estadísticamente significativas entre las variables dependientes involucradas en el modelo, se analizaron por grupo antes de la intervención mediante una prueba de análisis de varianza (ANOVA). Fue empleado un análisis de prueba T pareada para constatar diferencias en las variables dependientes antes y después de la intervención. Por último, el modelo explicativo se generó mediante el empleo de ecuaciones estructurales. Se contemplaron como criterios de ajuste los estadísticos $\chi^{2} / \mathrm{df}$, NFI, RFI, IFI, TLI, CFI y RMSEA siguiendo las recomendaciones de Byrne (2010). Los valores contemplados fueron: $\chi^{2} / \mathrm{df}<2$; normed-fit index (NFI), relative fit index 
(RFI), incremental fit index (IFI), Tucker Lewis index (TLI) y comparative-fit index (CFI), mayor a ,09 y root-mean-square-error of approximation (RMSEA) menor a ,08 como valor razonable. Todos los análisis se realizaron mediante SPSS y AMOS (IBM), ambos en su versión 21.

\section{Resultados}

Los resultados de la confiabilidad obtenidos en las escalas empleadas para medir las variables dependientes estrés percibido, emociones positivas y emociones negativas se presentan en la Tabla 2. Como se aprecia contaron con un valor adecuado para su uso. En el caso de la escala $\mathrm{AGH}$, esta tuvo un $\alpha=, 85$

Tabla 2

Confiabilidad de Cronbach

\begin{tabular}{|l|l|l|}
\hline Escala & Pre Intervención & $\begin{array}{l}\text { Post Interven- } \\
\text { ción }\end{array}$ \\
\hline Estrés percibido (PSS) &, 84 &, 81 \\
\hline Subescala emociones Positivas (PANAS) &, 88 &, 90 \\
\hline Subescala emociones Negativas (PANAS) &, 84 &, 85 \\
\hline Escala de emociones (PANAS) &, 70 &, 79 \\
\hline
\end{tabular}

El análisis con la prueba ANOVA para identificar diferencias significativas entre los seis grupos, antes de la intervención, para el valor de variables dependientes fueron: emociones positivas, $F(5,210)=, 703, \mathrm{p}=, 622$; emociones negativas, $F(5,210)=1,01, \mathrm{p}=, 410$; y estrés percibido, $F(5,210)=1,54, p=, 177$. Lo que corroboró la similitud entre estas a través de los grupos.

El resultado de la prueba T para grupos dependientes de las variables de estudio, realizado tras la intervención, se muestra en la Tabla 3. Se constata con ella que las medidas de interés mostraron una disminución significativa tras la implementación de la actividad.

Tabla 3

Cambio en las variables de emociones y estrés percibido tras realizar la actividad artística

\begin{tabular}{|l|l|l|l|l|l|}
\hline & \multicolumn{2}{|l|}{ Pre Intervención } & \multicolumn{2}{l|}{ Post Intervención } & Prueba T \\
\cline { 1 - 4 } & Media & D.E. & Media & D.E. & \\
\hline $\begin{array}{l}\text { Subescala emociones } \\
\text { positivas (PANAS) }\end{array}$ & 33,36 & 7,50 & 31,64 & 8,90 & $\begin{array}{l}\mathrm{T}(215)=3,57, \\
p=, 001, d=, 20\end{array}$ \\
\hline $\begin{array}{l}\text { Subescala emociones } \\
\text { negativas (PANAS) }\end{array}$ & 22,88 & 7,39 & 16,34 & 6,82 & $\begin{array}{l}\mathrm{T}(215)=13,96, \\
p=, 001, d=, 92\end{array}$ \\
\hline $\begin{array}{l}\text { Escala Estrés Percibido } \\
\text { (PSS) }\end{array}$ & 25,05 & 7,20 & 22,05 & 8,64 & $\begin{array}{l}\mathrm{T}(215)=6,60, \\
p=, 001, d=, 378\end{array}$ \\
\hline
\end{tabular}


Por último, el modelo de ecuaciones estructurales encontrado con adecuado ajuste a los datos se muestra en la Figura 4. Este representa la manera como la actividad artística permite afrontar las situaciones que se perciben como estresantes gracias a sus facultades como "Estrategia centrada en la solución de problemas" (F1); "Placer y valor terapéutico" (F2) y "Competencia y uso del material" (F3). como se observa, el impacto del estrés percibido sobre la actividad artístico-recreativa realizada para afrontar los estresores (afrontamiento) es alta y negativa $(-, 86)$ al ser evaluada antes de la intervención. Tras ella, la percepción del estrés disminuye, pero continúa siendo negativa (-,77). Es hasta esta segunda evaluación donde es posible encontrar una relación entre el estrés percibido y la valoración de las emociones negativas $(-, 45)$. Los resultados del análisis estadístico de este modelo figuran en la Tabla 4, donde se constata que los valores seleccionados cuentan con el ajuste adecuado.

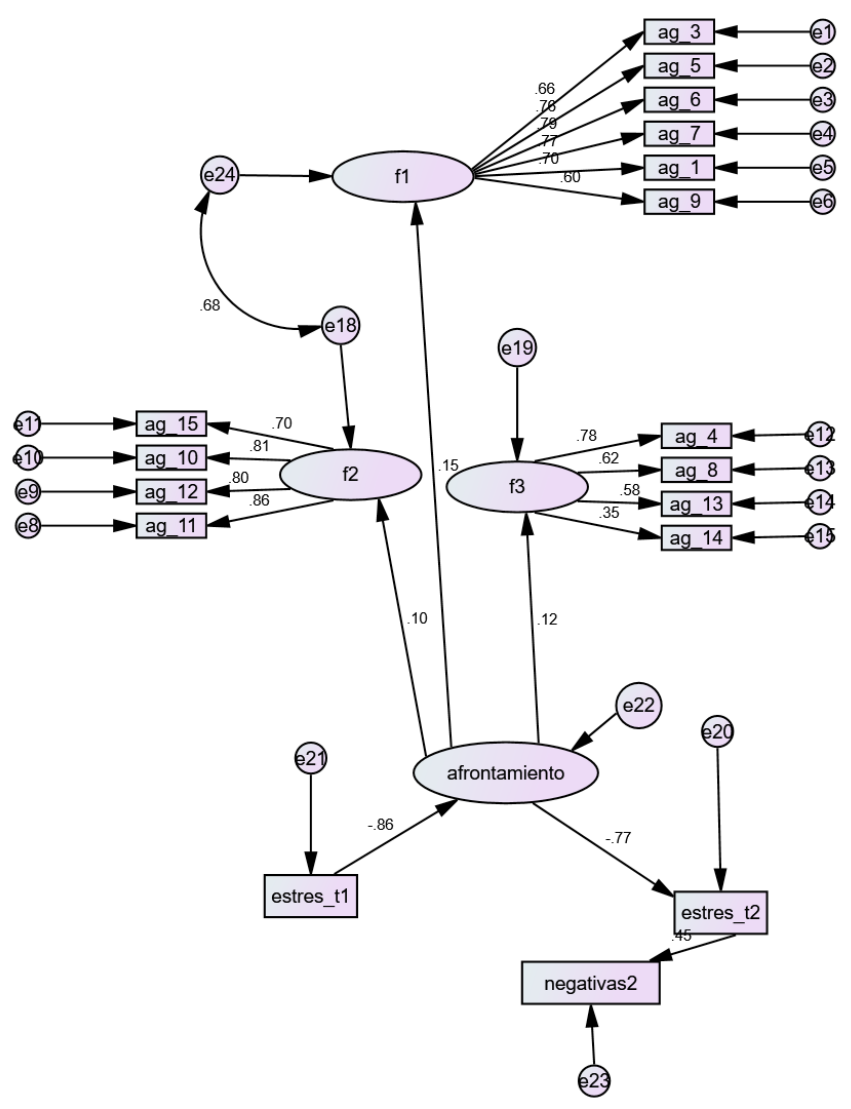

Figura 1. Mediación del estrés e impacto en las emociones tras utilizar el dibujo como herramienta una herramienta para el afrontamiento.

Nota: (estrés_t1) Estrés percibido pre intervención; (F1) Estrategia centrada en la solución de problema; (F2) Placer y valor terapéutico; (F3) Competencia y uso del material; (estrés_t2) Estrés percibido post intervención; (negativas2) Emociones negativas post intervención. 
Tabla 4

Índices de bondad de ajuste para el modelo estructural.

\begin{tabular}{|l|l|l|l|l|l|l|l|}
\hline Modelo & $\begin{array}{l}\text { CMIN/ } \\
\text { DF }\end{array}$ & NFI & RFI & IFI & TLI & CFI & $\begin{array}{l}\text { RM- } \\
\text { SEA }\end{array}$ \\
\hline $\begin{array}{l}\text { Modelo Hipo- } \\
\text { tético }\end{array}$ & 1,961 &, 951 &, 887 &, 976 &, 941 &, 975 &, 067 \\
\hline
\end{tabular}

\section{Discusión}

Este estudio tuvo como objetivo profundizar el análisis del manejo del estrés percibido y las emociones, a través de actividades artísticas. Para ello se evaluó la relación entre los aspectos cognitivos y las emociones de los participantes al realizar una actividad gráfica encaminada a disminuir el estrés cotidiano.

Siguiendo a Huss et al. (2012) y Drake \& Winner (2012a, 2012b), las actividades artísticas pueden ser usadas como un mediador que permite ya sea expresar o distraerse de los estresores. Nuestros resultados indican que ambos usos dan pie a la disminución tanto de las emociones como del estrés percibido.

Tras obtener resultados acordes con lo presenciado en otras investigaciones, como un segundo paso se procedió a diseñar un modelo explicativo que incluyese los cambios en el estrés y las emociones, y su relación con la experiencia artística. Esta parte del proceso, no sólo se sustenta en el resultado experimental obtenido, sino que también hace eco en los estudios empíricos documentados por Dilawari y Tripathi (2014) y Malchiodi (2005); quienes señalan que las actividades artísticas pueden aliviar el estrés cotidiano pues permiten que las personas se centren en el problema o bien se alejen de este, mediante una actividad que a su vez permite experimentar placer.

El modelo documentado en esta investigación no sólo toma en cuenta el placer generado por la actividad artística, también considera el valor de la competencia en el uso del material y el rol que juegan las emociones en dicho proceso. De esta manera, fue posible ubicar que, la actividad artística sí posibilita la disminución del estrés y las emociones negativas tras realizar un dibujo, tanto si se orienta a la distraerse de los estresores como si se busca enfocarse en soluciones sobre esto, dentro de sesiones de aproximadamente 30 minutos y en un ambiente experimental.

Este estudio difiere de otros por su manejo de la actividad artística para apoyar la salud psicológica pues indaga en el aporte de la técnica, y la relación que establece el usuario con esta, contemplando tanto el placer como la competencia que percibe de si mismo el usuario (Mercer et al., 2010; Regehr et al., 2013; Visnola et al., 2010). Es también distinto por trabajarse desde un enfoque cuantitativo y con un diseño de estudio que permite la manipulación de las variables, lo que hace más distinguible el aporte de cada factor involucrado; sin embargo, esta misma selección de diseño presenta a su vez la limitante de no permitir la observación en el ambiente natural del fenómeno. Y el limitar la generalización de los resultados a poblaciones con características similares.

Dadas estas implicaciones proponemos: replicar el estudio, incluyendo muestras de poblaciones distintas. Indagar el efecto de este tipo de actividades en las emocio- 
nes positivas, las cuales disminuyeron tras la intervención como las otras variables, cambio que puede interpretarse desde diferentes puntos de vista, pero que requieren de mayor investigación para ser explicado. En resumen, este estudio confirma estadísticamente la relación de algunos de los elementos involucrados en la valoración de la actividad artística como una herramienta capaz de disminuir el estrés cotidiano.

\section{Referencias}

Aydın, K., B. (2010). Strategies for coping with stress as predictors of mental health. International Journal of Human Sciences [Online]. 7:1. Available: http://www.insanbilimleri. com/en

Bitonte, R. \& De Santo, M. (2014). Art therapy: an underutilized yet effective tool. Mental Illness, 6, 18-19.

Cano, F., Rodríguez, L. \& García, J. (2007). Adaptación española del inventario de estrategias de afrontamiento. Acta Española de Psiquiatría, 35(1), 29-39. Recuperado de: http:// personal.us.es/fjcano/drupal/?q=node/8

Cohen, S., Kamarck, T. \& Mermelstein, R. (1983). A global measure of perceived stress. Journal of Health and Social Behavior, 24, 385-396.

Drake, J. \& Hodge, A. (2015). Drawing versus writing: The role of preference in regulation short-term affect. Art Therapy: Journal of the American Art Therapy Association, 32(1), 27-33.

Drake, J., Coleman, K. \& Winner, E. (2011). Short-term mood repair through art: Effects of medium and strategy. Art Therapy: Journal of the American Art Therapy Association, 28(1), 26-3

Drake, J. \& Winner, E. (2012a). Confronting sadness through art-making: Distraction is more beneficial than venting. Psychology of Aesthetics, Creativity and the Arts, 6(3), 255-261.

Drake, J. \& Winner, E. (2012b). How children use drawing to regulate their emotions. Cog-

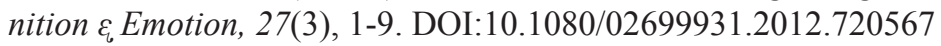

Dilawari, K. \& Tripathi N. (2014). Art therapy: A creative and expressive process. Indian Journal of Positive Psychology, 5(1), 81-85.

Fredrickson, B. \& Joiner, T. (2002). Positive emotions trigger upward spirals toward emotional well-being. American Psychological Society, 13(2), 172-175.

González, M. T. \& Landero, R. (2007). Factor Structure of the Perceived Stress Scale (PSS) in a Sample from Mexico. The Spanish Journal of Psychology, 10(1), 199-206.

González-Ramírez, M., Rodrígez-Ayán, M. \& Landero-Hernández, R. (2013). The perceived stress scale (pss): Normative data and factor structure for a large-sample in Mexico. The Spanish Journal of Pyschology, 16, E47. DOI:10.1017/sjp.2013.35.

Hacking, S., Secker, J., Spandler, H., Kent, L. \& Shenton, J. (2008). Evaluating the impact of participatory art projects for people with mental health needs. Health and Social Care in the Community, 16(6), 638-648. DOI:10.1111/j.1365-2524.2008. 00789.x

Holmqvist, G. \& Lundqvist, P. (2012). Is there evidence for the use of art therapy in treatment of psychosomatic disorders, eating disorders and crisis? A comparative study of two different systems for evaluation. Scandinavian Journal of Psychology, 53, 47-53. DOI: 10.1111/j.1467-9450.2011. 00923.x

Huet, V. \& Holttum, S. (2016). Art therapy-based groups for work-related stress with staff in health and social care: An exploratory study. The Arts in Psychotherapy, 50, 46-57. 
Huss, E. (2012). Integrating strengths and stressors through combining dynamic phenomenological and social perspectives into art evaluations. The Arts in Psychotherapy, 39, 451-455.

Huss, E., Nuttman-Shwartze, O. \& Altman, A. (2012). The role of collective symbols as enhancing resilience in children's art. The Arts in Psychotherapy, 39, 52-59. DOI: 10.1016/j. aip.2011.11005

Kim, S. (2013). A randomized, controlled study of the effects of art therapy on older Korean-Americans' healthy aging. The Arts in Psychotherapy, 40, 158-164. DOI: 10.1016/j. aip.2012.11002

Lang, P.J., Bradley, M.M. \& Cuthbert, B.N. (2008). International affective picture system (IAPS): Affective ratings of pictures and instruction manual. Technical Report A-8. University of Florida, Gainesville, FL

Lazarus, R. (2000). Estrés y emociones. Manejo e implicaciones en nuestra salud. España: Descleé de Brouwer.

Malchiodi, C. (2005). Expressive Therapies. En C. M. (editor), Expressive Therapies, Nueva York, Estados Unidos: Guilford Publication.

Marxen, E. (2011). Diálogos entre arte y terapia. Del "arte psicótico" al desarollo de la arteterapia y sus aplicaciones. Barcelona, España: Gedisa.

Mercer, A., Warson, E. \& Zhao, E. (2010). Visual journaling: An intervention to influence stress, anxiety and effect levels in medical students. The Arts in Psychotherapy, 37, 143148. doi:10.1016/j.aip.2009.12.003

Metzl, E. (2008). Systematic analysis of art therapy research published in Art Therapy: Journal of AATA between 1987 and 2004. The Arts in Psychotherapy, 35(1), 60-73.

Mische, L., Williams, P., Glennon, C., Carithers, K., Schnabel, E. Andrejack, A. \& Wright N. (2012). Effect of art making on cancer-related symptoms of blood and marrow transplantion recipients. Oncology Nursing Forum, 39(4), 353-360.

O'Brien, A., Terry, D. \& Jimmieson, N. (2008). Negative affectivity and responses to work stressors: An experimental study. Anxiety, Stress and Coping, 21(1), 55-83. doi:10.1080/10615800701529504

Regehr, C., Glancy, D. \& Pitts, A. (2013). Interventions to reduce stress in university students: A review and meta-analysis. Journal of Affective Disorders, 15, 148(1): 1-11. DOI: 10.1016/j.jad.2012.11.026

Reynolds, F. (2010). 'Colour and communion': Exploring the influences of visual art-making as a leisure activity on older women's subjective well-being. Journal of Aging Studies, 24, 135-143. DOI: 10.1016/j.jaging.2008.10.004

Reynolds, M., Nabors, L. \& Quinlan, A. (2000). The effectiveness of art therapy: does it work? Art Therapy: Journal of the American Art Therapy Association, 17(3), 207-213.

Sarid, O. \& Huss, E. (2010). Trauma and acute stress disorder: A comparision between behavioral intervention and art therapy. Arts in Psycotherapy, 37, 8-12. DOI: 10.1016/j. aip.2009.11.004

Singer, S., Götze, H., Buttstädt, M., Geue, K., Momenghalibaf, A. \& Böhler, U. (2010). The effects of an art education program on competencies, coping, and well-being in outpatients with cancer: Results of a prospective feasibility study. The Arts in Psychotherapy, 37, 363-369. DOI: 10.1016/j.aip.2010.07.002

Slayton, S., D'Archer, J. \& Kaplan, F. (2010). Outcomes studies on the efficacy of art therapy: A review of findings. Art Therapy: Journal of the American Art Therapy Association, 27(3), 108-118. DOI:10.1080/07421656.2010.10129660 
Snir, S. \& Regev, D. (2013). ABI- Art-based Intervention questionnaire. The Arts in Psychoterapy, 40, 338-346. DOI: 10.1016/j.aip.2013.06.005

Vanegas-Farfano, M., Quezada-Berumen, L. y González Ramírez, M. Actividades artístico-recreativas y estrés percibido. (2016). Ansiedad y Estrés.

Visnola, D., Sprūdža, D., Baķe, M. \& Piķe, A. (2010). Effects of art therapy on stress and anxiety of employees. Proceedings of the Latvian Academy of Sciences, 64, 85-91. doi: 10.2478/v10046-010-0020-y

Watson, D., Clark, L. A. \& Tellegen, A. (1988). Development and validation of brief measures of positive and negative affect: The PANAS scales. Journal of Personality and Social Psychology, 54, 1063-1070. 